\title{
Review of neurocysticercosis
}

\author{
Julio Sotelo, M.D., ANd Oscar H. Del Brutto, M.D. \\ National Institute of Neurology and Neurosurgery, Mexico City, Mexico; and Department of \\ Neurological Sciences, Hospital-Clínica Kennedy, Guayaquil, Ecuador
}

\begin{abstract}
In the neurosurgical services in many developing countries, treatment of neurocysticercosis (NCC) accounts for greater than $10 \%$ of brain surgical procedures and approximately $15 \%$ of neurological consultations. In these areas brain cysticercosis is the leading cause of hydrocephalus in adults and the first cause of late-onset epilepsy. During the last two decades, successful medical treatment has been established. Additionally, neuroimaging and immunological studies have clearly defined the topography, pathophysiological mechanisms, and biological status of these lesions. Thus, selection of cases for medical or surgical treatment has improved; in a significant number of cases, both interventions are required. New therapies with either albendazole or praziquantel have respectively reduced to 8 days and to 1 day the course of anticysticidal therapy, which now is fast, effective, inexpensive, atoxic, and convenient, particularly in endemic areas where most patients belong to the lower socioeconomic groups. Additionally, the rational use of steroid agents facilitates the treatment of inflammation, a conspicuous accompaniment in cases of NCC. A major effort, however, is still required to eradicate this disease.
\end{abstract}

\section{KEY WORD • neurocysticercosis • hydrocephalus • epilepsy}

Neurocysticercosis is caused by the larvae of the tapeworm Taenia solium in the nervous system, a disease suffered by millions of people living in the developing countries of Central and South America, sub-Saharan Africa, and east and south Asia..$^{7,9,26,47}$ In these areas, the disease accounts for up to $12 \%$ of all admissions to neurological hospitals and is the leading cause of acquired epilepsy in adults. ${ }^{34,42}$ More than 50,000 new NCC-related deaths occur annually, and of the many more patients who suffer related neurological sequelae, most are affected at productive ages. ${ }^{24,53}$ This makes NCC a large public health problem in developing countries. Moreover, massive emigration of people from endemic to nonendemic areas has increased its prevalence in North America and some European countries, where this condition had been considered rare. ${ }^{57,76}$

\section{ETIOPATHOGENESIS OF NEUROCYSTICERCOSIS}

Taenia solium has a complex life cycle that requires two hosts. Humans are the only known hosts for the adult ces-

Abbreviations used in this paper: $\mathrm{CNS}=$ central nervous system; $\mathrm{CSF}=$ cerebrospinal fluid; $\mathrm{CT}=$ computerized tomography; ELISA = enzyme-linked immunosorbent assay; EITB = enzymelinked immunoelectrotransfer blot; $\mathrm{MR}=$ magnetic resonance; $\mathrm{NCC}=$ neurocysticercosis. tode, whereas both pigs and humans may act as intermediate hosts for the larval form or cysticercus. ${ }^{30}$ The adult T. solium inhabits the small intestine in humans, where it is attached to the intestinal wall by its suckers and hooks. Every day, a few pregnant proglottids are detached from the distal end of the body of the worm and passed with the feces; each proglottid contains thousands of eggs, which are fully embryonated, infective, and resistant to adverse environments. In regions in which systems for disposal of human feces are deficient, wandering pigs eat human feces containing $T$. solium eggs. Once in the intestinal tract of the pig, the action of bile and pancreatic enzymes cause the eggs to lose their coats, liberating oncospheres that cross the intestinal wall, enter the bloodstream, and are carried to the tissues of the host where embryos evolve to form larvae (cysticerci). In these circumstances, pigs become the intermediate hosts in the life cycle of $T$. solium.

Human consumption of improperly cooked infected pork results in the transmission of viable cysticerci in the small intestine, where, by the action of bile and digestive enzymes, the scolex of a cysticercus evaginates and attaches to the intestinal wall. Thereafter, proglottids begin to multiply and the parasite becomes a cestode that can be passed in the feces as mature proglottids approximately 4 months after attachment. Humans can also act as intermediate hosts for T. solium after ingestion of Taenia eggs. In these circumstances, human cysticercosis develops. ${ }^{24,30}$ The mechanisms by which eggs cross the intestinal wall 


\section{J. Sotelo and O. H. Del Brutto}

and lodge in human tissues are the same as those in pigs. The two main sources from which humans contact cysticercosis are ingestion of food contaminated with $T$. solium eggs and the fecal-oral route in individuals harboring the intestinal cestode.

Cysticerci are vesicles consisting of two main parts: the vesicular wall and the scolex. The scolex has a similar morphology to the adult $T$. solium, including an armed rostellum and a rudimentary body ${ }^{45}$ The macroscopic appearance of cysticerci varies according to their location within the CNS. Viable cysticerci in brain parenchyma measure approximately $10 \mathrm{~mm}$. These cysts tend to lodge in the cerebral cortex or the basal ganglia because of the high vascular supply in these areas. Subarachnoid cysticerci may be located within cortical sulci or in the CSF cisterns at the base of the brain. ${ }^{3}$ After a long period of infection some cysticerci lose their scolices and grow to reach $50 \mathrm{~mm}$ or more, which more frequently occurs in cysts located in the subarachnoid cisterns, because their growth is not limited by the effect of pressure within the brain parenchyma. Some of these cysts are composed of several membranes attached to each other with a grapelike appearance (racemose form of cysticerci) ${ }^{27}$ Ventricular cysts are usually single lesions; they may be attached to the choroid plexus or float freely within the ventricular cavities. Other locations within the CNS include the subdural space, the sellar region, the eye, and the spinal cord. ${ }^{1,6,20}$

After entering the CNS, cysticerci are viable and induce slight inflammatory changes in the surrounding tissues. At this stage, called the vesicular stage, parasites have a transparent membrane, clear vesicular fluid, and larva or scolex. They may remain for decades in this stage or, as the result of a complex immunological attack from the host, may begin a degenerative process that may lead to the death of the parasite and its further transformation into an inert nodule. ${ }^{27}$ After cysticidal treatment or successful immune response, the first sign of involution of cysticerci is the colloidal stage, in which the transparent vesicular fluid is replaced by a viscous and turbid fluid, readily identifiable by MR imaging studies, ${ }^{55}$ and the scolex dies. Colloidal cysticerci are surrounded by a thick collagen capsule, and the surrounding brain parenchyma shows an intense astrocytic gliosis associated with microglial proliferation, diffuse edema, neuronal degenerative changes, and perivascular cuffing of lymphocytes. Thereafter, the wall of the cyst thickens and the scolex is transformed into coarse mineralized granules; this stage, in which the cysticercus is not longer viable, is called the granular nodular stage. Finally, in the calcified stage the parasite remnants appear as a mineralized (calcified) nodule. When parasites enter into the granular and calcified stages, the edema subsides but the astrocytic changes in the vicinity of the lesions are conspicuous. The exact duration of each of these stages in the natural history of the infection has not been established, because considerable differences exist among individuals, particularly in relation to the intensity of the endogenous immune response. Moreover, the authors of histopathological studies have demonstrated cysticerci in different stages of involution in the same individual, suggesting that the immune reaction from the host in response to cysticerci infection of the CNS is heterogeneous. ${ }^{30}$

Meningeal cysticerci elicit an intense inflammatory re- action in the subarachnoid space, forming an exudate composed of collagen fibers, lymphocytes, multinucleated giant cells, eosinophils, and hyalinized parasitic membranes leading to abnormal thickening of the leptomeninges and difficulty in CSF absorption, which produces hydrocephalus in a significant number of patients. ${ }^{3,45}$ The optic chiasm and cranial nerves arising from the anterior aspect of the brainstem are encased in this leptomeningeal thickening. Because the foramina of Luschka and Magendie may also be occluded by the thickened leptomeninges and parasitic membranes, noncommunicating hydrocephalus subsequently may develop. ${ }^{29}$ Small penetrating arteries arising from the circle of Willis are frequently affected by the subarachnoid inflammatory reaction elicited by meningeal cysticerci, and this may cause occlusion of the lumen of the vessel and the subsequent development of cerebral infarctions. ${ }^{13,43}$

Ventricular cysticerci also cause an inflammatory reaction if they are attached to the choroid plexus or the ventricular wall. The ependymal lining is disrupted, and ependymal cells are replaced by proliferating subependymal glial cells that protrude toward the ventricular cavities and may block the transit of CSF, particularly when the site of protrusion is at or near the foramen of Monro or the cerebral aqueduct. This process is called granular ependymitis and is usually associated with hydrocephalus. ${ }^{2}$

Cysticerci have several antigens that stimulate the production of specific antibodies, which can be detected on immunodiagnostic tests. Some antigens may play a role in the mechanisms by which cysticerci evade the immune response. ${ }^{24}$ One of them, antigen $\mathrm{B}$, may be localized both within the parasite and in the surrounding tissue. It is partly composed of collagen and may bind to C1q, inhibiting the classic pathway of complement activation. Because destruction of cysticerci appears to be mediated by activation of the complement cascade, antigen B could play a role in the protection of cysticerci against the host's immunological attack. ${ }^{77}$ Analysis of several studies suggests the presence of cellular immune dysfunction in patients with NCC. This depressed immunity may be responsible for the reported association of this parasitic disease with conditions resulting from immunological disturbances such as hematological malignancies and conditions leading to the development of cerebral tumors. ${ }^{18,19,38}$

\section{CLINICAL MANIFESTATIONS}

Neurocysticercosis may affect males and females from birth through advanced age; however, the peak age of its incidence is in middle-aged adults. The disease may present with a variety of clinical manifestations, a pleomorphic quality that is related to individual differences in the number and location of the lesions within the CNS. ${ }^{3,8,16}$, $58,61,66$ This fact underscores the lack of specificity in the disease's clinical picture and the need to undertake complementary studies in every patient to confirm its diagnosis. 21,63

Epilepsy occurs in up to $70 \%$ of patients with NCC and is its most common clinical manifestation. ${ }^{22}$ In most of these cases neurological status is normal, and their signs differ from those with other forms of acquired epilepsy who usually present with focal neurological signs. Neurocysticercosis-induced seizures are most commonly gener- 
alized tonic-clonic or simple partial, although some patients may present with complex partial seizures. ${ }^{22,42}$

A variety of focal neurological findings related to the size, number, and location of the parasites have been described in patients with NCC. Whereas pyramidal tract signs predominate, almost every focal sign that may occur in disorders of the CNS has been described in NCC. Focal neurological signs usually follow a subacute or chronic course that resembles that of a brain tumor. Some patients, however, present with rapid development of focal signs due to the occurrence of a cerebrovascular event. ${ }^{13,52}$ Other patients present with increased intracranial pressure and dementia signs. Hydrocephalus, related to cysticercal arachnoiditis, granular ependymitis, or ventricular cysts, is the most common cause of this syndrome. ${ }^{29}$ Increased intracranial pressure also occurs in patients with giant cysts and in those with cysticercal encephalitis. The latter manifestation is a severe form of the disease, more common in children and young women, that occurs as the result of a massive cysticercal infection of the brain parenchyma inducing an intense immune response and diffuse brain edema. ${ }^{50}$

A number of patients with NCC present with intellectual impairment; ${ }^{31}$ in the past, prior to the advance of neuroimaging studies, some of these individuals were admitted to psychiatric hospitals for several years until the correct diagnosis was suspected after the occurrence of seizures or focal neurological signs. Patients with sellar region cysticerci present with ophthalmological and endocrinological disturbances similar to those produced by pituitary tumors or craniopharyngiomas. ${ }^{20}$ In cases of spinal cord cysticercosis, patients present with nonspecific clinical manifestations such as nerve root pain or sensorimotor deficits that vary according to the level of the lesion. ${ }^{1}$ Intraocular cysticerci cause progressive decrease of visual acuity or visual field defects. ${ }^{6}$ Massive cysticercal infection of striated muscles occasionally produces a clinical picture of generalized weakness associated with muscle pseudohypertrophy. ${ }^{56}$

\section{LABORATORY STUDIES}

The frequency with which $T$. solium eggs are detected in stool examinations in patients with NCC varies according to the severity of infection. Indeed, the high prevalence of taeniasis found in cases of massive cysticercal infection of the CNS suggests that autoinfection occurred in most of these patients by the fecal-oral route. ${ }^{33,36}$ It is also important to search for $T$. solium carriers among the close relatives of these patients. Recognition of Taenia eggs is not easy, and infection in many patients may escape detection when only a single coproparasitological study is performed. Serial stool specimens must be examined before the patient is considered to be free of infection. ${ }^{51}$ Two recent advances may improve the diagnosis of human taeniasis: ELISA for coproantigens detection and DNA hybridization for egg identification. ${ }^{76}$

Abnormalities in the cytochemical composition of CSF have been reported in as many as $80 \%$ of patients with active NCC. These abnormalities correlate with the activity of the disease and with the location of parasites within the CNS; therefore, CSF examination yielding no evidence of infection does not rule out the diagnosis of
NCC. ${ }^{24}$ The most consistent finding is moderate mononuclear pleocytosis, with cell counts rarely exceeding 300/ $\mathrm{mm}^{3}$; however, as many as 5000 cells $/ \mathrm{mm}^{3}$ may be present in patients with severe cysticercal meningitis. Elevated protein levels are common in patients with pleocytosis; protein levels are usually raised within the range of 50 to $300 \mathrm{mg} / \mathrm{dl}$, although they have also been reported as high as $1600 \mathrm{mg} / \mathrm{dl}$. Cerebrospinal fluid glucose levels are usually within the normal range despite active meningeal disease; however, hypoglycorrhachia is present in up to $18 \%$ of NCC patients, and low glucose levels have been associated with poor prognosis.

Immunodiagnostic tests of serum samples have been widely used to assess the prevalence of cysticercosis and to exclude or confirm the diagnosis of NCC in patients with neurological signs but in whom neuroimaging findings are inconclusive. Because the accuracy of these tests relies on the highly variable humoral immune response of the host against cysticerci, however, they have unsatisfactory sensitivity and specificity. False-negative results are related to local production of antibodies within the CNS without a parallel increase of antibodies in peripheral blood, or to immune tolerance to the parasite without production of anticysticercal antibodies; they are mostly the result of previous contact with the parasite or to cross reactivity with other helminths, which is particularly frequent in individuals from endemic areas.

The complement fixation test was initially developed to determine the presence of anticysticercal antibodies in serum; however, its performance is better when applied to CSF samples. ${ }^{32}$ It is positive in $83 \%$ of patients in whom inflammatory changes occurred in the CSF but only in $22 \%$ of those with parenchymal cysticercosis in whom normal cytochemical analysis of CSF was demonstrated. This is an important shortcoming because many patients with NCC CSF abnormalities are not detected on this analysis. The complement fixation test is also less sensitive in ventricular than in subarachnoid CSF. ${ }^{55}$

The ELISA has been widely used. In as many as $30 \%$ of patients with NCC, however, a false-negative serum ELISA may occur, and a false-positive result may be demonstrated in a similar number of patients because of cross reactivity with other infectious diseases. ${ }^{49}$ The ELISA performed to test CSF samples is more accurate than that for serum; its sensitivity is $87 \%$ and its specificity is $95 \% .{ }^{54}$ As occurs when conducting the complement fixation test, these results depend on the presence of active disease, because many patients with parenchymal brain calcifications or granulomas, the ELISA demonstrates no abnormal findings even if the test is performed in CSF samples.

When it was found that antibodies to specific antigens of $T$. solium can be detected by EITB assay, investigators were impelled to develop highly purified antigens of cysticercus to be used in this new immunological diagnostic test. Seven antigenic bands are usually recognized by antibodies found in patients with cysticercosis. Among these, GP13, GP14, GP24, and GP39-42 are the most frequently recognized antigens. ${ }^{74}$ The EITB has been extensively evaluated in different hospital- and population-based studies. $^{34,44}$ Whereas the authors of some reports indicate that serum EITB is highly sensitive and specific for the diagnosis of human cysticercosis, results reported by others have been disappointing. ${ }^{44}$ One shortcoming of the test is 
the high incidence of false-negative results in patients harboring a single cerebral cyst; in such cases, the sensitivity of EITB decreases to $30 \%$. In EITB, an assay conducted in patients with calcified lesions is less likely to demonstrate a positive result than in those with active disease. Another weakness of the serum EITB is that the test may be positive in patients with taeniasis. This is an important shortcoming in the accuracy of the test when attempting to determine the prevalence of human cysticercosis in endemic areas, because many patients exposed to the adult parasite may test positive. The results of EITB must be interpreted in light of the clinical manifestations, neuroimaging findings, and more importantly, the geographic origin of the patient. A positive EITB result is of lesser diagnostic value in patients from areas where cysticercosis is endemic. A negative EITB result does not exclude a diagnosis of NCC in patients harboring a single cerebral lesion or in those with parenchymal brain calcifications as the sole evidence of the disease.

\section{NEUROIMAGING STUDIES}

Both CT and MR imaging have drastically improved diagnostic accuracy for NCC by providing objective evidence concerning the topography of the lesions and the degree of the host's inflammatory response against the parasite. ${ }^{37,40,55,72}$ The CT and MR imaging findings in patients with parenchymal brain cysticercosis depend on the developmental stage of the cysticerci. Characteristic neuroimaging features include the scolex appearing as a hyperintense nodule ("hole-with-dot" imaging) as well as punctuate calcifications. ${ }^{72}$ In contrast, single or multiple ringlike or nodular enhancing lesions, as seen in patients with cysticerci in the acute encephalitic phase or in those with cysticercal encephalitis, are nonspecific and represent a diagnostic challenge because pyogenic brain abscesses, fungal abscesses, tuberculomas, toxoplasma abscesses, and brain tumors may produce similar findings on CT or MR imaging. .,16,50,55

Computerized tomography and MR imaging findings in meningeal cysticercosis include hydrocephalus, abnormal enhancement of the leptomeninges at the skull base, large subarachnoid cysts, and cerebral infarcts. ${ }^{3,40}$ In patients with infarcts, cerebral angiography may reveal segmental narrowing or occlusion of major intracranial arteries. ${ }^{52}$ With the exception of cystic lesions, most of these findings are nonspecific and may also be observed in patients with tuberculous, sarcomatous, or fungal meningitis. Diagnostic cornerstones for differential diagnosis of these entities are neurological examination and results of CSF analysis. Patients with NCC rarely, if ever, suffer fever or signs of meningeal irritation, and CSF glucose content is usually in the normal range. ${ }^{24}$ In contrast, most other conditions producing subacute or chronic meningitis course with fever, neck stiffness, and decreased CSF glucose levels.

Ventricular cysticerci appear on neuroimaging studies as cystic lesions that distort the anatomy of the ventricular system and cause asymmetrical hydrocephalus. ${ }^{37,55}$ These lesions are usually isodense with CSF on MR imaging but are not well visualized on CT scanning. Therefore, CT scanning with administration of intraventricular contrast medium is usually needed to visualize the cysts. On MR imaging, however, most ventricular cysts are detected because the scolex is better delineated and because the signal properties of the cystic fluid and CSF are different on $\mathrm{T}_{2}$-weighted imaging sequences. . $^{37,40,55}$

In general, MR imaging is better than CT scanning for the diagnosis of NCC, particularly in patients with skull base lesions, brainstem cysts, intraventricular cysts, and spinal lesions. Nevertheless, an important shortcoming in the accuracy of MR imaging for the diagnosis of NCC is the detection of small calcifications. Because in many of these patients parenchymal calcifications are the sole evidence of the disease, their condition may not be detected by MR imaging. Computerized tomography scanning remains the best neuroimaging screening procedure in patients with suspected NCC; MR imaging may be reserved for patients in whom CT scanning demonstrates no evidence of these lesions or in whom CT findings are inconclusive..$^{55,61}$

\section{SURGICAL AND PHARMACOLOGICAL THERAPY}

To undertake the optimum intervention, NCC must be characterized in terms of the cyst's viability, degree of the host's immune response to the parasites, and location of the lesions. ${ }^{14,25,55}$ Therapy includes a combination of specific cysticidal drugs, noncysticidal therapy, and surgery.

\section{Cysticidal Drugs}

Praziquantel has been used to treat human NCC for nearly two decades. The authors of several studies have shown that praziquantel destroys as much as $70 \%$ of parenchymal brain cysticerci after a 15-day course of treatment at daily doses of $50 \mathrm{mg} / \mathrm{kg} .{ }^{65,70}$ This initial regimen was arbitrarily chosen. Since that time, recommended doses of praziquantel have ranged from 10 to 100 $\mathrm{mg} / \mathrm{kg}$ for periods of 3 to 21 days..$^{5}$ In these studies, praziquantel was administered every 8 hours. Because plasma levels of praziquantel decline in less than 3 hours after administration, it seems that the cysticidal effect is reached by exposing the parasites to several intermittent peaks of the drug. On this basis, it has been suggested that if parenchymal brain cysticerci are exposed to high concentrations of praziquantel maintained for up to 6 hours (three doses of $25-30 \mathrm{mg} / \mathrm{kg}$ at 2-hour intervals on a single day), this might be sufficient to destroy the parasites. ${ }^{10}$ Preliminary results are encouraging. The decrease in the number of cysts visualized on neuroimaging studies after a 1-day therapy is similar to that observed in patients receiving longer courses of the drug at conventional doses. ${ }^{10,17,39,46}$

Albendazole is another strong cysticidal drug, initially tested at doses of $15 \mathrm{mg} / \mathrm{kg} /$ day for a 1 -month period. ${ }^{28} \mathrm{It}$ was later found, however, that at similar doses the length of therapy could be shortened to 1 week without lessening the drug efficacy. ${ }^{35,68}$ Albendazole destroys 75 to $90 \%$ of parenchymal brain cysts and has been shown to be superior to praziquantel in several comparative trials. ${ }^{11,62,64,73}$ Another advantage of albendazole over praziquantel is that the former also destroys subarachnoid and ventricular cysts because of its better penetration in CSF, as well as the fact that it can be administered jointly with corticosteroid agents for antiinflammatory therapy. ${ }^{12,23,48}$ 
The introduction of praziquantel and albendazole has radically changed the prognosis in most patients with NCC. Results of the initial studies, however, generated skepticism regarding the integral benefit to the patient after the destruction of the parasites. More recently, investigators focusing on the long-term clinical outcome after treatment with cysticidal drugs have shown that cysticidal therapy also produces marked clinical improvement in most patients. ${ }^{25}$ The control of seizures with antiepileptic drugs in patients with epilepsy due to parenchymal brain cysts is much better after treatment with cysticidal drugs than when the disease is left untreated. ${ }^{22,41,75}$ Moreover, some authors have demonstrated that patients with focal neurological deficits have recovered after a trial with cysticidal drugs because the pressure effects exerted by the parasites against eloquent cerebral areas have been resolved as the result of therapy. ${ }^{23,55}$

Cysticidal treatment in patients with cysticercal encephalitis should be performed in conjunction with immunosuppressive therapy because the sudden destruction of a heavy parasite burden may exacerbate the intracranial hypertension observed in this form of the disease.$^{50} \mathrm{In}$ patients with both hydrocephalus and intracranial cysts, cysticidal drugs can be administered after a ventricular shunt has been placed to avoid further increase of the intracranial pressure as a result of drug therapy. Cysticidal drugs must be used with caution in patients with giant subarachnoid cysticerci because the inflammatory reaction occurring in the host in response to the destruction of parasites within the subarachnoid space may occlude small leptomeningeal vessels surrounding the cyst. In such patients, concomitant administration of steroid agent is mandatory to prevent a cerebral infarction. ${ }^{12,23,48}$ In patients with ventricular cysts, the therapeutic approach with albendazole drugs should be closely monitored. Finally, patients with calcifications alone do not require anticysticercal drugs because these lesions represent the sequelae of parasites previously eliminated.

\section{Noncysticidal Therapy}

Because inflammation is the conspicuous accompaniment of most forms of NCC, corticosteroids represent the primary form of therapy for cysticercal encephalitis, angiitis, and arachnoiditis causing hydrocephalus and progressive entrapment of cranial nerves. ${ }^{25}$ In such cases, up to $30 \mathrm{mg} /$ day of dexamethasone may be needed for control of symptoms. In patients with cysticercal encephalitis, corticosteroids may be used in association with mannitol at doses of $2 \mathrm{mg} / \mathrm{kg} / \mathrm{day}$. The initial trial with high doses of intravenous dexamethasone may be followed by chronic oral therapy with prednisone $(50 \mathrm{mg} /$ day) or dexamethasone $(10 \mathrm{mg} /$ day $)$ administered three times a week.

The simultaneous administration of corticosteroids and cysticidal drugs has been recommended to ameliorate the secondary effects of headache and vomiting that may occur during praziquantel or albendazole therapy. Such manifestations are not related to toxic effects of cysticidal therapy but to the acute destruction of parasites within the brain; thus, they are reliable indicators of drug efficacy. Common analgesic and antiemetic medications may be used to ameliorate such symptoms, avoiding the compul- sory use of corticosteroids in every patient. ${ }^{65}$ Absolute indications for corticosteroid administration during cysticidal drug therapy include the management of giant subarachnoid cysticerci, ventricular cysts, spinal cysts, and multiple parenchymal brain cysts. In these cases, corticosteroids must be administered before, during, and even some days after the course of anticysticercal drugs to avoid the risk of cerebral infarction, acute hydrocephalus, spinal cord swelling, and massive brain edema, respectively. ${ }^{25}$

In patients with calcified cysticerci-induced seizures, the administration of standard doses of a single, first-line antiepileptic drug usually results in adequate seizure control. In patients with parenchymal brain cysts cysticidal drugs must also be administered to achieve an adequate control of seizures with antiepileptic drugs. ${ }^{22,41,75}$ The optimum length of antiepileptic drug therapy in these patients, however, has not yet been determined. The authors of a prospective study have shown that up to $50 \%$ of patients suffered recurrences after cessation of antiepileptic therapy. ${ }^{15}$ These patients had been free of seizures for 2 years, and albendazole had successfully destroyed their brain cysts. In that report prognostic factors associated with seizure recurrence included the development of parenchymal brain calcifications as the result of albendazole therapy, as well as the development of both recurrent seizures and multiple brain cysts before the institution of anticysticercal therapy.

\section{Surgical Treatment}

At present, in most centers in which physicians have considerable experience in the treatment of NCC, extirpation of the cysticerci is limited only to those cases in which cysticidal treatment fails. Patients with hydrocephalus secondary to cysticercal arachnoiditis require the placement of a ventricular shunt; however, there is a high incidence of shunt dysfunction due mostly to the high content of cells and protein in the CSF. The protracted course in these patients and their high mortality rates $(\leq 50 \%$ in 2 years) are directly related to the number of surgical interventions necessary to revise the shunt. ${ }^{67}$ Chronic corticosteroid administration has been suggested to reduce the risk of shunt occlusion. ${ }^{71}$ In addition, a new shunt device functioning at a constant flow without a valve mechanism has been recently developed. ${ }^{59,69}$ This shunt does not allow the entrance of spinal CSF into the ventricular system toward the shunt device. In patients with NCC, this inversion of CSF transit is the most common cause of shunt dysfunction because it allows subarachnoid inflammatory cells and activated immune cells to enter the ventricular cavities. Long-term results obtained when using this new shunt are encouraging. ${ }^{60}$

Ventricular cysts may be excised or endoscopically aspirated. ${ }^{4}$ There is, however, the possibility that a cyst may migrate between the time of diagnosis and surgery, and this phenomenon must be ruled out by obtaining a control CT or MR image immediately before surgery to avoid unnecessary craniotomies. ${ }^{78}$ In the absence of ependymitis, permanent shunt placement is usually not necessary after removal of a ventricular cyst. In contrast, shunt placement should precede the excision of ventricular cysts associated with ependymitis. 


\section{References}

1. Akiguchi I, Fujiwara T, Matsuyama H, et al: Intramedullary spinal cysticercosis. Neurology 29:1531-1534, 1979

2. Apuzzo MLJ, Dobkin WR, Zee CS, et al: Surgical considerations in treatment of intraventricular cysticercosis. An analysis of 45 cases. J Neurosurg 60:400-407, 1984

3. Bandres JC, White AC Jr, Samo T, et al: Extraparenchymal neurocysticercosis: report of five cases and review of management. Clin Infect Dis 15:799-811, 1992

4. Bergsneider M, Holly LT, Lee JH, et al: Endoscopic management of cysticercal cysts within the lateral and third ventricles. J Neurosurg 92:14-23, 2000

5. Bittencourt PRM, Gracia CM, Gorz AM, et al: High-dose praziquantel for neurocysticercosis: efficacy and tolerability. Eur Neurol 30:229-234, 1990

6. Cardenas F, Quiroz H, Plancarte A, et al: Taenia solium ocular cysticercosis: findings in 30 cases. Ann Ophthalmol 24: 25-28, 1992

7. Chandy MJ, Rajshekhar V, Ghosh S, et al: Single small enhancing CT lesions in Indian patients with epilepsy: clinical radiological and pathological considerations. J Neurol Neurosurg Psychiatry 54:702-705, 1991

8. Chang GY, Keane JR: Visual loss in cysticercosis: analysis of 23 patients. Neurology 57:545-548, 2001

9. Coker-Vann MR, Subianto DB, Brown P, et al: ELISA antibodies to cysticerci of Taenia solium in human populations in New Guinea, Oceania, and Southeast Asia. Southeast Asian J Trop Med Public Health 12:499-505, 1981

10. Corona T, Lugo R, Medina R, et al: Single-day praziquantel therapy for neurocysticercosis. N Engl J Med 334:125, 1996

11. Cruz M, Cruz I, Horton J: Albendazole vs praziquantel in the treatment of cerebral cysticercosis: clinical evaluation. Trans $\mathbf{R}$ Soc Trop Med Hyg 85:244-247, 1991

12. Del Brutto OH: Albendazole therapy for subarachnoid cysticerci: clinical and neuroimaging analysis of 17 patients. J Neurol Neurosurg Psychiatry 62:659-661, 1997

13. Del Brutto OH: Cysticercosis and cerebrovascular disease: a review. J Neurol Neurosurg Psychiatry 55:252-254, 1992

14. Del Brutto OH: Medical treatment of cysticercosis-effective. Arch Neurol 52:102-104, 1995

15. Del Brutto OH: Prognostic factors for seizure recurrence after withdrawal of antiepileptic drugs in patients with neurocysticercosis. Neurology 44:1706-1709, 1994

16. Del Brutto OH: Single parenchymal brain cysticercus in the acute encephalitic phase: definition of a distinct form of neurocysticercosis with benign prognosis. J Neurol Neurosurg Psychiatry 58:247-249, 1995

17. Del Brutto OH, Campos X, Sánchez J, et al: Single-day praziquantel versus 1-week albendazole for neurocysticercosis. Neurology 52:1079-1081, 1999

18. Del Brutto OH, Castillo PR, Mena IX, et al: Neurocysticercosis among patients with cerebral gliomas. Arch Neurol 54: 1125-1128, 1997

19. Del Brutto OH, Dolezal M, Castillo PR, et al: Neurocysticercosis and oncogenesis. Arch Med Res 31:151-155, 2000

20. Del Brutto OH, Guevara J, Sotelo J: Intrasellar cysticercosis. J Neurosurg 69:58-60, 1988

21. Del Brutto OH, Rajshekhar V, White AC Jr, et al: Proposed diagnostic criteria for neurocysticercosis. Neurology 57: 177-183, 2001

22. Del Brutto OH, Santibañez R, Noboa CA, et al: Epilepsy due to neurocysticercosis: analysis of 203 patients. Neurology 42: 389-392, 1992

23. Del Brutto OH, Sotelo J, Aguirre R, et al: Albendazole therapy for giant subarachnoid cysticerci. Arch Neurol 49:535-538, 1992

24. Del Brutto OH, Sotelo J, Román GC: Neurocysticercosis: A Clinical Handbook. Lisse, The Netherlands: Swets \& Zeitlinger, 1998
25. Del Brutto OH, Sotelo J, Roman GC: Therapy for neurocysticercosis: a reappraisal. Clin Infec Dis 17:730-735, 1993

26. Díaz F, García HH, Gilman RH, et al: Epidemiology of taeniasis and cysticercosis in a Peruvian village. The Cysticercosis Working Group in Peru. Am J Epidemiol 135:875-882, 1992

27. Escobar A, Nieto D: Parasitic diseases, in Minckler J (ed): Pathology of the Nervous System, Volume 3. New York: McGraw-Hill, 1972, pp 2503-2521

28. Escobedo F, Penagos P, Rodriguez J, et al: Albendazole therapy for neurocysticercosis. Arch Intern Med 147:738-741, 1987

29. Estañol B, Kleriga E, Loyo M, et al: Mechanisms of hydrocephalus in cerebral cysticercosis: implications for therapy. Neurosurgery 13:119-123, 1983

30. Flisser A: Taeniasis and cysticercosis due to Taenia solium. Prog Clin Parasitol 4:77-116, 1994

31. Forlenza OV, Filho AHGV, Nobrega JPS, et al: Psychiatric manifestations of neurocysticercosis: a study of 38 patients from a neurology clinic in Brazil. J Neurol Neurosurg Psychiatry 62:612-616, 1997

32. García E, Sotelo J: A new complement fixation test for the diagnosis of neurocysticercosis in cerebrospinal fluid. J Neurol 238:379-392, 1991

33. García HH, Del Brutto OH: Heavy nonencephalitic cerebral cysticercosis in tapeworm carriers. The Cysticercosis Working Group in Peru. Neurology 53:1582-1584, 1999

34. García HH, Gilman R, Martinez M, et al: Cysticercosis as a major cause of epilepsy in Peru. The Cysticercosis Working Group in Peru (CWG). Lancet 341:197-200, 1993

35. García HH, Gilman RH, Horton J, et al: Albendazole therapy for neurocysticercosis: a prospective double-blind trial comparing 7 versus 14 days of treatment. Cysticercosis Working Group in Peru. Neurology 48:1421-1427, 1997

36. Gilman RH, Del Brutto OH, García HH, et al: Prevalence of taeniosis among patients with neurocysticercosis is related to the severity of infection. The Cysticercosis Working Group in Peru. Neurology 55:1062, 2000

37. Ginier BL, Poirier VC: MR imaging of intraventricular cysticercosis. AJNR 13:1247-1248, 1992

38. Herrera LA, Ramirez T, Rodriguez U, et al: Possible association between Taenia solium cysticercosis and cancer: increased frequency of DNA damage in peripheral lymphocytes from neurocysticercosis patients. Trans R Soc Trop Med Hyg 94:61-65, 2000

39. López-Gómez M, Castro N, Jung H, et al: Optimization of the single-day praziquantel therapy for neurocysticercosis. Neurology 1929-1930, 2001

40. Martinez HR, Rangel-Guerra R, Elizondo G, et al: MR imaging in neurocysticercosis: a study of 56 cases. AJNR 10: 1011-1019, 1989

41. Medina MT, Genton P, Montoya MC, et al: Effect of anticysticercal treatment on the prognosis of epilepsy in neurocysticercosis: a pilot trial. Epilepsy 34:1024-1027, 1993

42. Medina MT, Rosas E, Rubio-Donnadieu F, et al: Neurocysticercosis as the main cause of late-onset epilepsy in Mexico. Arch Intern Med 150:325-327, 1990

43. Monteiro L, Almeida-Pinto J, Leite I, et al: Cerebral cysticercus arteritis: five angiographic cases. Cerebrovasc Dis 4:125-133, 1994

44. Ordoñez G, Medina MT, Sotelo J: Immunoblot analysis of serum and CSF from patients with various forms of neurocysticercosis. Neurol Infect Epidemiol 1:57-61, 1996

45. Pittella JEH: Neurocysticercosis. Brain Pathol 7:681-693, 1997

46. Pretell EJ, García HH, Custodio N, et al: Short regimen of praziquantel in the treatment of single brain enhancing lesions. Clin Neurol Neurosurg 102:215-218, 2000

47. Preux PM, Melaku Z, Druet-Cabanac M, et al: Cysticercosis and neurocysticercosis in Africa: current status. Neurol Infect Epidemiol 1:63-68, 1996

48. Proaño JV, Madrazo I, Avelar F, et al: Medical treatment for 
neurocysticercosis characterized by giant subarachnoid cysts. $\mathbf{N}$ Engl J Med 345:879-885, 2001

49. Ramos-Kuri M, Montoya RM, Padilla A, et al: Immunodiagnosis of neurocysticercosis: disappointing performance of serology (enzyme-linked immunosorbent assay) in an unbiased sample of neurological patients. Arch Neurol 49:633-636, 1992

50. Rangel R, Torres B, Del Bruto O, et al: Cysticercotic encephalitis: a severe form in young females. Am J Trop Med Hyg 36: 387-392, 1987

51. Richards F Jr, Schantz PM: Laboratory diagnosis of cysticercosis. Clin Lab Med 11:1011-1028, 1991

52. Rodriguez-Carbajal J, Del Brutto OH, Penagos P, et al: Occlusion of the middle cerebral artery due to cysticercotic angiitis. Stroke 20:1095-1099, 1989

53. Roman G, Sotelo J, Del Brutto O, et al: A proposal to declare neurocysticercosis an international reportable disease. Bull World Health Organ 78:399-406, 2000

54. Rosas N, Sotelo J, Nieto D: ELISA in the diagnosis of neurocysticercosis. Arch Neurol 43:353-356, 1986

55. Salgado P, Rojas R, Sotelo J: Cysticercosis. Clinical classification based on imaging studies. Arch Intern Med 157: 1991-1997, 1997

56. Sawhney BB, Chopra JS, Banerji AK, et al: Pseudohypertrophic myopathy in cysticercosis. Neurology 26:270-272, 1976

57. Shandera WX, White AC Jr, Chen JC, et al: Neurocysticercosis in Houston, Texas. A report of 112 cases. Medicine 73:37-52, 1994

58. Sotelo J: Cysticercosis, in Vinken PJ, Bruyn GW, Klawans HL (eds): Handbook of Clinical Neurology. Amsterdam: Elsevier, 1988, Vol 8, pp 529-534

59. Sotelo J: A new ventriculoperitoneal shunt for treatment of hydrocephalus. Experimental results. RBM 15:257-262, 1993

60. Sotelo J: Update: the new ventriculoperitoneal shunt at The Institute of Neurology of Mexico. Surg Neurol 46:19-20, 1996

61. Sotelo J, Del Brutto OH: Brain cysticercosis. Arch Med Res 31:3-14, 2000

62. Sotelo J, del Brutto OH, Penagos P, et al: Comparison of therapeutic regimen of anticysticercal drugs for parenchymal brain cysticercosis. J Neurol 237:69-72, 1990

63. Sotelo J, Del Brutto OH, Román GC: Cysticercosis. Curr Clin Top Infect Dis 16:240-259, 1996

64. Sotelo J, Escobedo F, Penagos P: Albendazole vs praziquantel therapy for neurocysticercosis. A controlled trial. Arch Neurol 45:532-534, 1988

65. Sotelo J, Escobedo F, Rodriguez-Carbajal J, et al: Therapy of parenchymal brain cysticercosis with praziquantel. N Engl J Med 310:1001-1007, 1984
66. Sotelo J, Guerrero V, Rubio F: Neurocysticercosis: a new classification based on active and inative forms. A study of 753 cases. Arch Intern Med 145:442-445, 1985

67. Sotelo J, Marin C: Hydrocephalus secondary to cysticercotic arachnoiditis. A long-term follow-up review of 92 cases. J Neurosurg 66:686-689, 1987

68. Sotelo J, Penagos P, Escobedo F, et al: Short course of albendazole therapy for neurocysticercosis. Arch Neurol 45: 1130-1133, 1988

69. Sotelo J, Rubalcava MA, Gomez-Llata S: A new shunt for hydrocephalus that relies on CSF production rather than on ventricular pressure: initial clinical experiences. Surg Neurol 43: 324-332, 1995

70. Sotelo J, Torres B, Rubio-Donnadieu F, et al: Praziquantel in the treatment of neurocysticercosis: a long-term follow-up. Neurology 35:752-755, 1985

71. Suastegui Roman RA, Soto-Hernández JL, Sotelo J: Effects of prednisone on ventriculoperitoneal shunt function in hydrocephalus secondary to cysticercosis: a preliminary study. J Neurosurg 84:629-633, 1996

72. Suss RA, Maravilla KR, Thompson J: MR imaging of intracranial cysticercosis: comparison with CT and anatomopathologic features. AJNR 7:235-242, 1986

73. Takayanagui OM, Jardim E: Therapy for neurocysticercosis. Comparison between albendazole and praziquantel. Arch Neurol 49:290-294, 1992

74. Tsang VCW, Brand JA, Boyer AE: An enzyme-linked immunoelectrotransfer blot assay and glycoprotein antigens for diagnosing human cysticercosis (Taenia solium). J Infect Dis 159: 50-59, 1989

75. Vasquez N, Sotelo J: The course of seizures after treatment for cerebral cysticercosis. N Engl J Med 327:696-701, 1992

76. White AC Jr: Neurocysticercosis: updates on epidemiology, pathogenesis, diagnosis, and management. Annu Rev Med 51: 187-206, 2000

77. White AC Jr, Tato P, Molinari JL: Host-parasite interactions in Taenia solium cysticercosis. Infect Agents Dis 1:185-193, 1992

78. Zee CS, Segall HD, Apuzzo MLJ, et al: Intraventricular cysticercal cysts: further neuroradiologic observations and neurosurgical implications. AJNR 5:727-730, 1984

Manuscript received April 15, 2002.

Accepted in final form May 16, 2002.

Address reprint requests to: Julio Sotelo, M.D., National Institute of Neurology and Neurosurgery of Mexico, Insurgentes Sur 3877, Mexico City, 14269, Mexico. email: jsotelo@servidor.unam.mx. 